Check for updates

Cite this: J. Mater. Chem. A, 2017, 5 , 22495

Received 29th September 2017 Accepted 17th October 2017

DOI: $10.1039 / \mathrm{c} 7 \mathrm{ta} 08617 \mathrm{~b}$

rsc.li/materials-a

\section{Understanding the stability of mixed A-cation lead iodide perovskites $\dagger$}

\author{
Bethan Charles, Jessica Dillon, Oliver J. Weber, M. Saiful Islam (D)* \\ and Mark T. Weller (DD*
}

The routes and kinetics of the degradation of thin films of methylammonium (MA)/formamidinium (FA) lead iodide perovskites $\left(\mathrm{MA}_{1-x} \mathrm{FA}_{x} \mathrm{Pbl}_{3}, 0 \leq x \leq 1\right)$ under dry atmospheric conditions have been investigated. MA-rich phases decompose to the precursor iodide salts and $\mathrm{Pbl}_{2}$, while $\mathrm{FA}$-rich phases convert mainly to the yellow hexagonal phase. The reactivity is strongly inhibited for mixed cation phases of $\mathrm{MA}_{1-x} \mathrm{FA}_{x} \mathrm{Pbl}_{3}$, for $x=0.4$ to 0.6 , where the decomposition routes available to end member phases become less favourable. It is shown that for pristine films with $x=0.6, \mathrm{Pbl}_{2}$ formation can be completely suppressed for up to 10 days. Kinetic analysis reveals that the rate of $\mathrm{Pbl}_{2}$ formation decays exponentially with increasing FA content until $x=0.7$, beyond which the FA containing perovskite transforms rapidly to the hexagonal phase. Ab initio simulations of the decomposition reaction energies fully support the increased kinetic stability found experimentally for the mixed A-cation perovskites.

Hybrid lead halide perovskites have recently risen to prominence as highly versatile materials for optoelectronic technologies, particularly high efficiency, solution processed solar cells. ${ }^{1-4}$ They display near optimal band gap for solar light absorption, high absorption coefficients, steep absorption onsets and significant charge carrier lifetimes (and thus diffusion lengths). ${ }^{2,5}$ These properties, combined with the processability of these materials, ${ }^{6,7}$ have resulted in solar cell device efficiencies rising rapidly to over $22 \%{ }^{8}$ However, the commercial applicability of these materials is hampered by their relative lack of stability compared to established inorganic and organic semiconductors. ${ }^{9-11}$ By chemical site-substitution at any of the $\mathrm{A}, \mathrm{B}$ or $\mathrm{X}$ sites of the $\mathrm{ABX}_{3}$ perovskite structure, it has been found that it is possible to tune the properties and, most pertinently, the stability of the resultant material. ${ }^{12-14}$

Centre for Sustainable Chemical Technologies, Department of Chemistry, University of Bath, Bath, BA2 7AY, UK.E-mail:m.t.weller@bath.ac.uk; m.s.islam@bath.ac.uk

$\dagger$ Electronic supplementary information (ESI) available: Synthesis and characterisation methods, SEM micrographs, additional PXD patterns and kinetic analysis. Experimental and $a b$ initio simulation methods. See DOI: $10.1039 / \mathrm{c} 7 \mathrm{ta0} 08617 \mathrm{~b}$
The archetypal hybrid perovskite methylammonium (MA) lead iodide $\left(\mathrm{CH}_{3} \mathrm{NH}_{3} \mathrm{PbI}_{3}\right)$, MAPI, crystallises in the tetragonal space group $14 / \mathrm{mcm}$ at room temperature. ${ }^{15}$ Formamidinium (FA) lead iodide $\left(\mathrm{CH}\left(\mathrm{NH}_{2}\right)_{2} \mathrm{PbI}_{3}\right)$, FAPI, by contrast preferentially crystallises in the yellow, $2 \mathrm{H}$ hexagonal perovskite type polymorph at room temperature, which can be driven to transform to the black cubic perovskite polymorph (required for photovoltaic applications) by heating above room temperature. ${ }^{16-19}$ Such varying phase behaviour can be accounted for by the different sizes and hydrogen bonding mechanisms of these dissimilar organic cations, MA and FA.

Both MA and FA cations display directional hydrogen bonding, a factor of increasing importance in controlling phase behaviour as the system temperature is reduced and also for defining the relative stability of the two polymorphs of FAPI. ${ }^{20}$ Stabilisation of mixed A site compounds relative to the end members of the series can be rationalised using geometrical arguments in which the perovskite tolerance factor is tuned using differently sized cations, as well as an entropic factor due to cation mixing. ${ }^{21}$

It has been established that by combining the MA and FA cations in solid solutions, the cubic perovskite phase is stabilised versus decomposition. ${ }^{22-25}$ We have previously shown that for compositions $\mathrm{MA}_{1-x} \mathrm{FA}_{x} \mathrm{PbI}_{3} 0<x<0.2$ the structures are best described by a tetragonal unit cell from $\mathrm{X}$-ray diffraction, while the rest of the composition range, $0.2 \leq x \leq 1$, forms with a cubic cell, space group $P m \overline{3} m{ }^{26}$

The compositional space for A site substitution between FA and MA has been explored in the literature. ${ }^{27,28}$ Huang et al. ${ }^{23}$ studied the charge transport characteristics of single $\mathrm{MA}_{1-x} \mathrm{FA}_{x} \mathrm{PbI}_{3}$ single crystals over the composition ranges $0<x<0.2$ and $0.8<x<1$ in increments of $x=0.05$. High carrier mobilities and lifetimes were obtained for $x=0.1$ and 0.15 . Promising performance has also been demonstrated recently in photovoltaic devices, ${ }^{22,29}$ where $\mathrm{MA}_{0.9} \mathrm{FA}_{0.1} \mathrm{PbI}_{3}$ delivered an efficiency improvement to $20.2 \%$, versus $18.6 \%$ for pure MAPI. Links between crystallinity, crystal phase stability, good optoelectronic properties and operational stability have been 
drawn, ${ }^{24}$ indicating that highly crystalline compositions also suppress effects such as mixed halide phase segregation.

Furthermore, for the synthesis and efficient operation of devices the perovskite materials need to be stabilised with respect to the phase transition to the undesirable hexagonal $2 \mathrm{H}-$ perovskite polymorph, as occurs for FAPI and FA-rich solid solutions. ${ }^{23,25}$ The phase stability of $\mathrm{MA}_{1-x} \mathrm{FA}_{x} \mathrm{PbI}_{3}$ has been studied for samples stored under a nitrogen atmosphere, indicating that for FA-rich compositions with $x>0.76$, partial transition to the hexagonal phase is observed within days, while for compositions with more MA, the hexagonal phase is not observed by X-ray diffraction. ${ }^{30}$ The propensity for hybrid perovskites to react with water has also been reported. ${ }^{31}$ While high photovoltaic performance for $\mathrm{MA}_{1-x} \mathrm{FA}_{x} \mathrm{PbI}_{3}$ has been demonstrated, the extended stability lifetime and decomposition of these compounds under moderately dry atmospheric conditions, likely to be similar to those used in device processing, have yet to be fully reported and are not completely understood.

In this work, for the first time, the degradation of $\mathrm{MA}_{1-x} \mathrm{FA}_{x} \mathrm{PbI}_{3}$ thin films over the full compositional range $0<x<1$, in increments of $x=0.1$, in air has been systematically investigated. The results provide a full understanding of the processes, thermodynamics and kinetics involved in the degradation of mixed A-cation lead perovskites.

Powder X-ray diffraction (PXD) data from $\mathrm{MA}_{1-x} \mathrm{FA}_{x} \mathrm{PbI}_{3}$ were initially collected on as-made pristine films (see ESI S1 and S2 $\dagger$ ). Further diffraction patterns were subsequently obtained from the same samples after 1, 3, 5, 7 and 10 days. The films were stored below $30 \%$ relative humidity in the dark in order to control exposure to high levels of moisture and intense light, which are already known to cause degradation. ${ }^{31}$ Raw diffraction data were corrected for sample height displacement and analysed using the EVA diffraction suite.

PXD patterns for samples with $0 \leq x \leq 1$ displayed the emergence of a peak centred at $2 \theta=12.7^{\circ}$, representing the (001) reflection for $\mathrm{PbI}_{2}$ (Fig. 1). For $0 \leq x \leq 0.3$, this reflection grew in intensity over time, surpassing the relative intensity of the (100) perovskite reflection centred at $2 \theta=14^{\circ}$ after 10 days. However, consideration of PXD data from samples with $x \geq 0.4$ showed that the presence of increasing levels of FA cation suppress the formation of $\mathrm{PbI}_{2}$, with the relative intensity of the (001) $\mathrm{PbI}_{2}$ reflection decreasing with increasing $x$, for the same

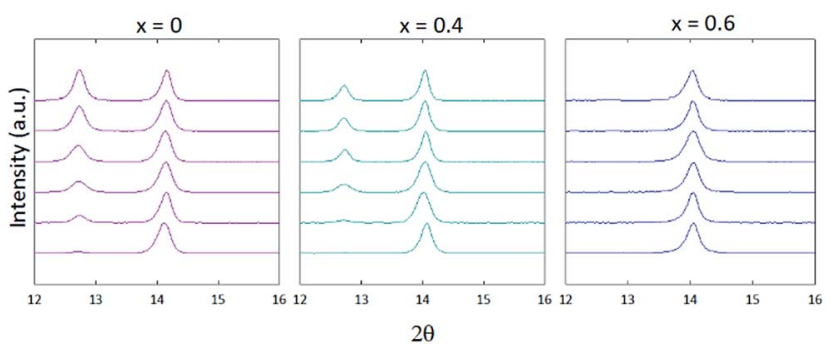

Fig. 1 Powder $\mathrm{X}$-Ray patterns of $\mathrm{MA}_{1-x} \mathrm{FA}_{x} \mathrm{Pbl}_{3}$ thin films where $x=0$, 0.4 and 0.6 . Patterns were measured immediately after synthesis (bottom trace) then through days 1, 3, 5, 7 and 10 (top trace). time period of exposure to air. For the film composition $x=0.6$, no $\mathrm{PbI}_{2}$ is observed after 7 days by PXD, with only trace amounts present after 10 days.

For compositions $x \geq 0.7$, the hexagonal $\delta$-phase is observed immediately after film fabrication. As previously observed for FAPI, increasing the annealing temperature to $180^{\circ} \mathrm{C}$ achieves full conversion to the perovskite $\alpha$-phase for $\mathrm{MA}_{1-x} \mathrm{FA}_{x} \mathrm{PbI}_{3}$, $x \geq 0.8{ }^{32}$ However, these films convert back to the hexagonal $\delta$-phase within 30 minutes of exposure to air. Suppression of $\delta$-phase formation was observed for $x=0.7$.

The presence of small amounts of $\mathrm{PbI}_{2}$ in the as-made films was found, for several values of $x$, to cause markedly increased rates of degradation. This was investigated further through the synthesis of $x=0.6$ films that contained varying amounts of $\mathrm{PbI}_{2}$ in the as-made thin films. PXD analysis of these films over a 10 day period following exposure to air showed the intensity of the $\mathrm{PbI}_{2}$ (001) reflection grew most rapidly for samples that contained observable levels of $\mathrm{PbI}_{2}$ in the as-made sample (see ESI S3 $†$ ). It is apparent from the data that phase pure perovskite films are required to suppress the growth rates of $\mathrm{PbI}_{2}$, as even trace amounts of $\mathrm{PbI}_{2}$ present in as-made films markedly catalyse the rate of further $\mathrm{PbI}_{2}$ formation. At the low moisture level used in this study neither sunlight nor laboratory lighting had a discernible effect on the rate of material degradation.

The kinetics of the decomposition of thin films of $\mathrm{MA}_{1-x} \mathrm{FA}_{x} \mathrm{PbI}_{3} 0.0 \leq x \leq 0.6$ were investigated through analysis of the rate of $\mathrm{PbI}_{2}$ phase formation. This was modelled through the following Johnson-Mehl-Avrami-Kolmogorov (JMAK) equation,

$$
\alpha=1-\exp \left(-(k t)^{m}\right)
$$

where $\alpha$ is the phase fraction of the product, $k$ is the rate constant, $t$ is time and $m$ is the Avrami exponent, the value of which depends on the crystallisation mechanism. ${ }^{33}$ Phase fractions in the degrading films were initially estimated using Rietveld profile fitting of the PXD data. However, refinements for these highly textured orientated films resulted in phase fraction values with large errors. Therefore, the peak area $(A)$ of the (001) $\mathrm{PbI}_{2}$ reflection was used to approximate the extent of phase formation $(\alpha)$ as required for this analysis of the kinetics.

From the JMAK equation a linear relationship is expected when plotting $\ln (t)$ with $\ln \ln (1 / 1-A)$ (Fig. 2). For $x=0.0-0.3$ this linear relationship was observed up to 5 days. After this time, with very high levels of $\mathrm{PbI}_{2}$ present, the change in the peak area of the (001) $\mathrm{PbI}_{2}$ reflection is unlikely to be representative of the true phase fraction of $\mathrm{PbI}_{2}$ present in the surface of the film. For $x \geq 0.4$, with the lower levels of $\mathrm{PbI}_{2}$ formed, the linearity was observed for the full duration of the 10 day study. Extracting the gradient and intercept of the linear model for each composition, $x$, a trend in rate constant modelled to a simple exponential decay can be observed (Fig. 3). It should be noted these results do not give absolute values for the rate constant of $\mathrm{PbI}_{2}$ formation, due to the peak area approximation employed to yield the level of $\mathrm{PbI}_{2}$. Nevertheless, the exponential decay seen in Fig. 3 demonstrates a clear trend of increasing 


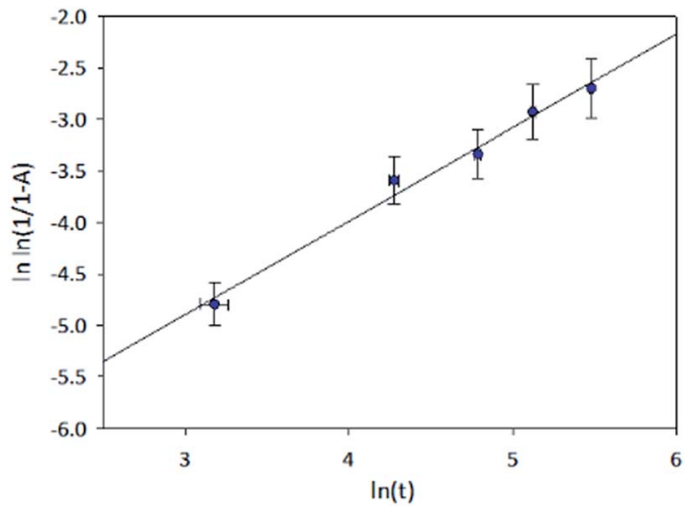

Fig. 2 Example Avrami plot for $\mathrm{MA}_{0.5} \mathrm{FA}_{0.5} \mathrm{Pbl}_{3}$. A linear relationship is observed when plotting $\ln (t)$ in hours and $\ln \ln (1 / 1-A)$, where the $A$ is peak area under the $\mathrm{Pbl}_{2}$ (001) reflections in the $\mathrm{X}$-ray diffraction patterns and $t$ is time in hours. Similar plots were produced for each value of $x$

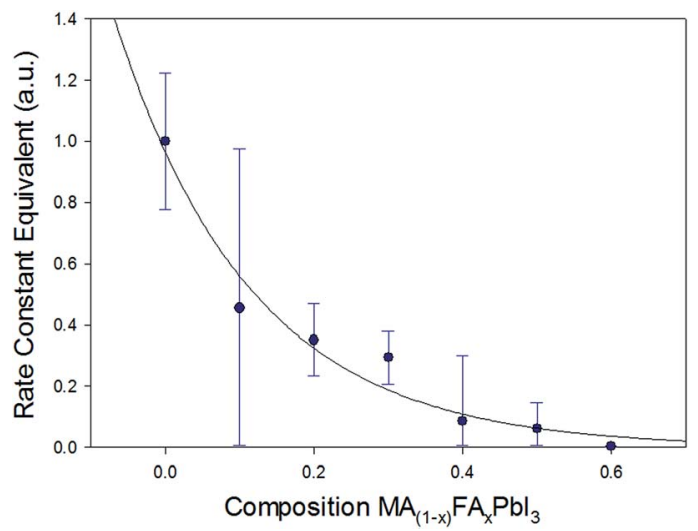

Fig. 3 Rate constant equivalents for $\mathrm{Pbl}_{2}$ growth fitted using an exponential decay function, calculated by extracting the intercept and gradient of linear models in the JMAK kinetic plots (Fig. 2), plotted using the peak area of the (001) $\mathrm{Pbl}_{2}$ reflection as an approximation for the phase fraction of $\mathrm{Pbl}_{2}$ transformed.

resistance in $\mathrm{MA}_{1-x} \mathrm{FA}_{x} \mathrm{PbI}_{3}$ film degradation to $\mathrm{PbI}_{2}$ as $x$ increases from 0.0 and approaches 0.6.

The degradation routes observed for $\mathrm{MA}_{1-x} \mathrm{FA}_{x} \mathrm{PbI}_{3}$ are summarised in Fig. 4. High rates of $\mathrm{PbI}_{2}$ formation are found for $x \leq 0.3$, although increasing the FA content strongly suppresses this decomposition pathway. For $x \geq 0.7$ this decomposition route competes with a phase transformation to the hexagonal $\delta$-phase.

Morphology and crystallite size within the thin films are factors that might affect the degradation rate. Therefore SEM images were acquired for the as-prepared $\mathrm{MA}_{1-x} \mathrm{FA}_{x} \mathrm{PbI}_{3}$ films (ESI S5 $\dagger$ ). For films with $x$ increasing from 0.0 to 0.6 the morphology was very similar and the average crystallite size (a few microns) only increased slightly with increasing FA content. For films with $x=0.7$, which coincides with the appearance of a portion of the hexagonal $\delta$-phase, a very different crystallite morphology and much larger crystallite size was observed. Therefore it seems likely that thermodynamics, as opposed to

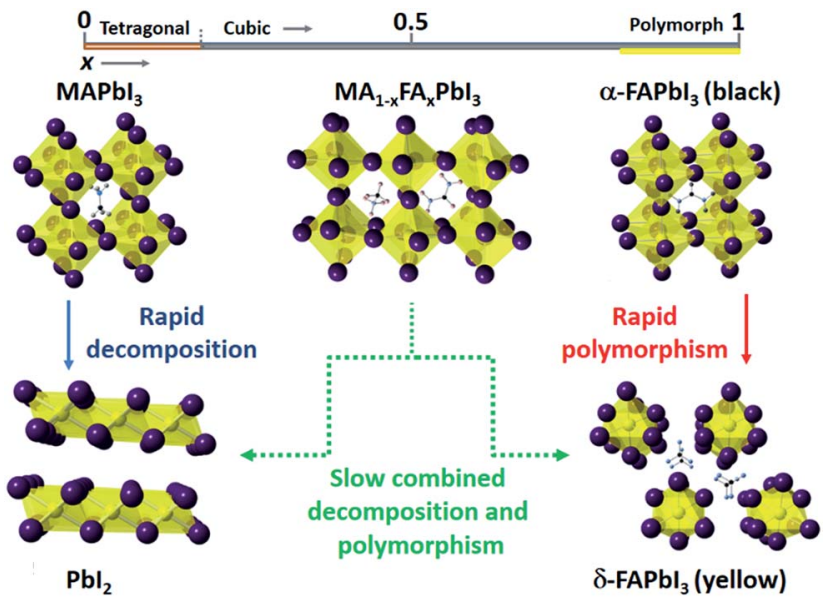

Fig. 4 Schematic of the experimentally determined degradation routes of $\mathrm{MA}_{1-x} \mathrm{FA}_{x} \mathrm{Pbl}_{3}(0 \leq x \leq 1)$. MA-rich phases decompose to $\mathrm{Pbl}_{2}$ and the precursor iodide salts, while FA-rich phases convert to the yellow $\delta$-phase. The routes are less favourable for the more stable $x=$ 0.4 to 0.6 mixed cation phases. $\mathrm{Pbl}_{6}$ octahedra are shown in yellow, with I represented as purple spheres.

film morphology, is a major factor contributing to the decomposition kinetics.

To complement our experimental diffraction and kinetic measurements, we performed $a b$ initio simulations on pure MAPI and a range of mixed MA/FA compositions to obtain insights into the trends in energetics of the degradation process. Such density functional theory (DFT) methods have been applied successfully to other recent studies on hybrid perovskites. $^{34-36}$

Using direct calorimetric techniques, Navrotsky et $a l .{ }^{37}$ have shown MAPI to be thermodynamically unstable with respect to decomposition to $\mathrm{PbI}_{2}$ and MAI. Here, the same intrinsic decomposition reaction was investigated involving the formation of $\mathrm{PbI}_{2}$ and organic iodides as well as decomposition to the $\delta$-phase:

$$
\mathrm{MA}_{1-x} \mathrm{FA}_{x} \mathrm{PbI}_{3} \rightarrow(1-x) \mathrm{MAI}+x \mathrm{FAI}+\mathrm{PbI}_{2}
$$

Other decomposition routes and the effect of the cubic to tetragonal phase transition for MA-rich phases are discussed further in the ESI S4. $\uparrow$ The reaction energies were calculated for a range of compositions $(x)$ and the values are presented in Fig. 5. The results reveal three main features.

First, pure MAPI shows a highly exothermic energy indicating thermodynamic instability with respect to the reaction products $\mathrm{PbI}_{2}$ and the organic iodide, which agrees well with experimental calorimetric measurements. ${ }^{37}$ Second, all the mixed $\mathrm{MA}_{1-x} \mathrm{FA}_{x} \mathrm{PbI}_{3}$ compositions $(0.0 \leq x \leq 0.6)$ show less favourable energetics, and hence a greater degree of stability, than pure $\mathrm{MAPbI}_{3}$. These results are consistent with our observation that formation of $\mathrm{PbI}_{2}$ was suppressed for increasing FA content with only trace amounts of $\mathrm{PbI}_{2}$ found after 10 days for $x=0.6$. We note that Brivio et al. ${ }^{38}$ find very small entropy terms for halide ion mixing in MAPI-based perovskites, which would further increase the stability of 


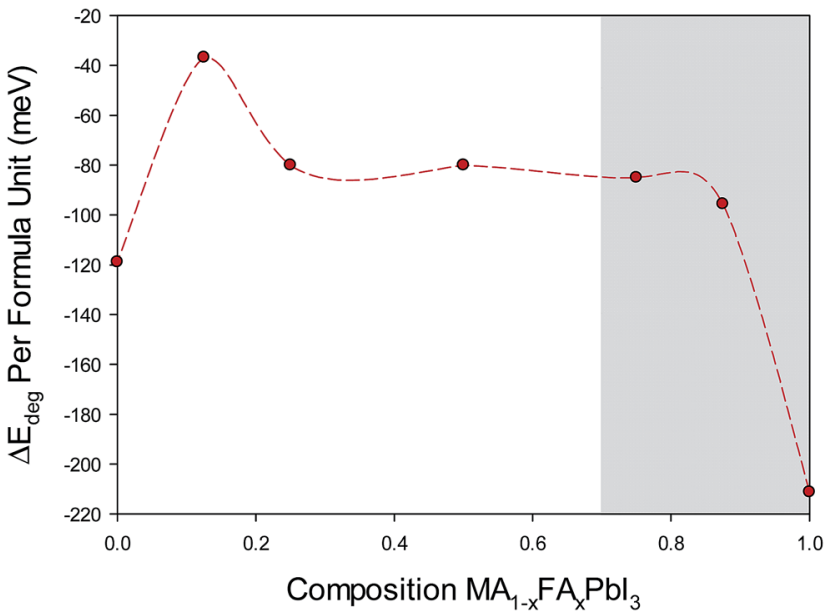

Fig. 5 Calculated energies for the decomposition reaction (2) for $\mathrm{MA}_{1-x} \mathrm{FA}_{x} \mathrm{Pbl}_{3}$ as a function of $\mathrm{FA}$ content $(x)$. For consistent comparison, starting structural configurations based on pseudo-cubic, high order structures are presented here (with full data showing the same trends found in ESI S4 ${ }^{\dagger}$ ). The shaded region indicates that in the preparation of compositions $x>0.7$ the yellow hexagonal (nonperovskite) polymorph is observed.

mixed cation compositions. Third, when we consider the decomposition reaction energy for perovskite-structured alpha$\mathrm{FAPbI}_{3}$ we find a highly exothermic energy suggesting a thermodynamically unstable structure. Indeed, it is known that in the preparation of compositions $x \geq 0.7$ the yellow hexagonal (non-perovskite) $\delta$-phase is observed at room temperature.

The kinetic and thermodynamic stability results are further supported through the theoretical optimum A-cation size suggested by the Goldschmidt and octahedral tolerance factors. Fig. 6 shows the effect of A site mixing on the Goldschmidt tolerance factor and octahedral factor, indicating which compositions are geometrically likely to adopt a cubic perovskite unit cell. Other well-known hybrid/inorganic halide

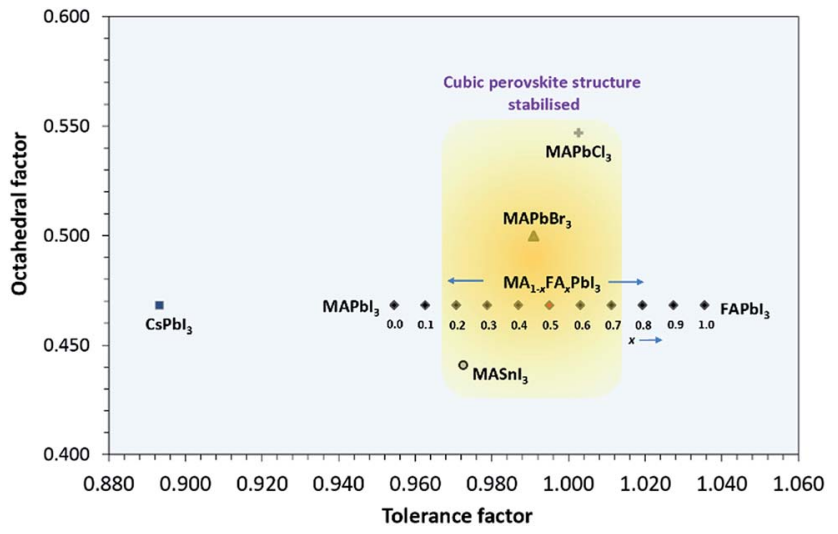

Fig. 6 Octahedral factor $(\mu)$ versus tolerance factor $(t)$ showing the range of compositions stabilised in the cubic perovskite structure for hybrid lead halide perovskites. Compositions $\mathrm{MA}_{1-x} \mathrm{FA}_{x} \mathrm{Pbl}_{3}, 0.3<x<$ 0.8 , which showed the greatest stability against degradation, are observed to lie close to the centre of the cubic perovskite structure stability field. perovskite compounds substituted at the A, B, or X sites are plotted for comparison. The octahedral factor is defined as:

$$
\mu=\frac{r_{\mathrm{B}}}{r_{\mathrm{X}}},
$$

And the Goldschmidt tolerance factor as:

$$
t=\frac{r_{\mathrm{A}}+r_{\mathrm{X}}}{\sqrt{2}\left(r_{\mathrm{B}}+r_{\mathrm{X}}\right)}
$$

where $r_{\mathrm{A}}, r_{\mathrm{B}}$ and $r_{\mathrm{X}}$ are the ionic radii of the $\mathrm{ABX}_{3}$ compound. Effective radii for organic cations taken from Kieslich et al. ${ }^{39}$ and revised ionic radii for iodide and bromide compounds are taken from Travis et al. ${ }^{40}$ Phases with the observed greatest stability towards decomposition, $\mathrm{MA}_{1-x} \mathrm{FA}_{x} \mathrm{PbI}_{3}, 0.3<x<0.8$, lie close to the centre of the cubic perovskite stability field.

In conclusion, thin films of the mixed organic cation perovskite system $\mathrm{MA}_{1-x} \mathrm{FA}_{x} \mathrm{PbI}_{3}$ undergo degradation in air to non-perovskite phases at a much slower rate than the endmember MAPI and FAPI phases. For example, the composition $\mathrm{MA}_{0.4} \mathrm{FA}_{0.6} \mathrm{PbI}_{3}$ synthesised with no contaminating $\mathrm{PbI}_{2}$ phase is stable in air for up to 10 days. Ab initio simulations of the decomposition reaction energies fully support the observed increased stability of the mixed A-cation perovskites. The reduced reactivity of these phases is likely to be related to suppression of the two possible degradation mechanisms of (i) decomposition to $\mathrm{PbI}_{2}$ and MAI, as found for MA-rich phases, and (ii) phase change as occurs for FA-rich phases. As FAPI does not degrade readily to FAI and $\mathrm{PbI}_{2}$, and MAPI adopts a single structure type, then any decomposition route of a mixed cation phase, such as $\mathrm{MA}_{0.4} \mathrm{FA}_{0.6} \mathrm{PbI}_{3}$, requires the simultaneous formation of three new structure types, namely yellow FAPI, $\mathrm{PbI}_{2}$ and MAI. We have shown that such a degradation route is less thermodynamically favourable and also has much slower kinetics. This combined experimental-computational study provides a greater understanding of the degradation routes of mixed A-cation perovskite halides, which is crucial for controlling and enhancing the long-term stability of perovskite solar cell materials.

\section{Conflicts of interest}

These is no conflict of interest to declare.

\section{Acknowledgements}

B. C. and O. J. W. thank the EPSRC for PhD studentship funding via the EPSRC CDT in Sustainable Chemical Technologies (EP/G03768X/1, EP/L016354/1). J. D. and M. S. I. acknowledge support from the University of Bath (URS PhD studentship) and the Materials Chemistry Consortium (EP/L000202) for Archer HPC facilities.

\section{Notes and references}

1 G. Xing, N. Mathews, S. Sun, S. S. Lim, Y. M. Lam, M. Grätzel, S. Mhaisalkar and T. C. Sum, Science, 2013, 342, 344-347. 
2 S. D. Stranks, G. E. Eperon, G. Grancini, C. Menelaou, M. J. P. Alcocer, T. Leijtens, L. M. Herz, A. Petrozza and H. J. Snaith, Science, 2013, 342, 341-344.

3 J. M. Ball, M. M. Lee, A. Hey and H. J. Snaith, Energy Environ. Sci., 2013, 6, 1739.

4 H. J. Snaith, J. Phys. Chem. Lett., 2013, 4, 3623-3630.

5 S. D. Stranks, V. M. Burlakov, T. Leijtens, J. M. Ball, A. Goriely and H. J. Snaith, Phys. Rev. Appl., 2014, 2, 34007.

6 M. T. Klug, A. Osherov, A. A. Haghighirad, S. D. Stranks, P. R. Brown, S. Bai, J. T.-W. Wang, X. Dang, V. Bulović, H. J. Snaith and A. M. Belcher, Energy Environ. Sci., 2017, 10, 236-246.

7 J. J. B. K. Xerxes Steirer, P. Schulz, G. Teeter, V. Stevanovic, M. Yang and K. Zhu, ACS Energy Lett., 2016, 1, 360-366.

8 M. A. Green, K. Emery, Y. Hishikawa, W. Warta, E. D. Dunlop, D. H. Levi and A. W. Y. Ho-Baillie, Prog. Photovolt. Res. Appl., 2017, 25, 3-13.

9 W. Rehman, D. P. McMeekin, J. B. Patel, R. L. Milot, M. B. Johnston, H. J. Snaith and L. M. Herz, Energy Environ. Sci., 2017, 10, 361-369.

10 H. Kim, J. Seo and N. Park, ChemSusChem, 2016, 9, 25282540.

11 D. Li, P. Liao, X. Shai, W. Huang, S. Liu, H. Li, Y. Shen and M. Wang, RSC Adv., 2016, 6, 89356-89366.

12 M. T. Klug, A. Osherov, A. A. Haghighirad, S. D. Stranks, P. R. Brown, S. Bai, J. T. Wang, X. Dang, V. Bulovic, H. J. Snaith and A. M. Belcher, Energy Environ. Sci., 2017, 10, 236-246.

13 C. C. Stoumpos, C. D. Malliakas and M. G. Kanatzidis, Inorg. Chem., 2013, 52, 9019-9038.

14 T. Leijtens, G. E. Eperon, N. K. Noel, S. N. Habisreutinger, A. Petrozza and H. J. Snaith, Adv. Energy Mater., 2015, 5, 1500963.

15 M. T. Weller, O. J. Weber, P. F. Henry, A. M. Di Pumpo and T. C. Hansen, Chem. Commun., 2015, 51, 4180-4183.

16 M. T. Weller, O. J. Weber, J. M. Frost and A. Walsh, J. Phys. Chem. Lett., 2015, 6, 3209-3212.

17 D. H. Fabini, C. C. Stoumpos, G. Laurita, A. Kaltzoglou, A. G. Kontos, P. Falaras, M. G. Kanatzidis and R. Seshadri, Angew. Chem., Int. Ed., 2016, 55, 15392-15396.

18 T. Chen, B. J. Foley, C. Park, C. M. Brown, L. W. Harriger, J. Lee, J. Ruff, M. Yoon, J. J. Choi and S.-H. Lee, Sci. Adv., 2016, 2(10), 1601650.

19 D. Araújo, M. J. Romero, F. Morier-Genoud and R. García, Mater. Sci. Eng., B, 1999, 66, 151-156.

20 G. Kieslich, S. Sun and A. K. Cheetham, Chem. Sci., 2014, 5, 4712-4715.

21 C. Yi, J. Luo, S. Meloni, A. Boziki, N. Ashari-Astani, C. Grätzel, S. M. Zakeeruddin, U. Rothlisberger and M. Grätzel, Energy Environ. Sci., 2015, 9, 656-662.
22 Y. Zhang, G. Grancini, Y. Feng, A. M. Asiri and M. K. Nazeeruddin, ACS Energy Lett., 2017, 2, 802-806.

23 Y. Huang, L. Li, Z. Liu, H. Jiao, Y. He, X. Wang, R. Zhu, D. Wang, J. Sun, Q. Chen and H. Zhou, J. Mater. Chem. A, 2017, 14, 38-44.

24 W. Rehman, D. P. McMeekin, J. B. Patel, R. L. Milot, M. B. Johnston, H. J. Snaith and L. M. Herz, Energy Environ. Sci., 2017, 131, 6050-6051.

25 A. Binek, F. C. Hanusch, P. Docampo and T. Bein, J. Phys. Chem. Lett., 2015, 6, 1249-1253.

26 M. T. Weller, O. J. Weber and B. Charles, J. Mater. Chem. A, 2016, 4, 15375-15382.

27 J. T. Jacobsson, J. P. Correa Baena, M. Pazoki, M. Saliba, K. Schenk, M. Grätzel and A. Hagfeldt, Energy Environ. Sci., 2016, 9, 1706-1724.

28 Z. Yang, C.-C. Chueh, P.-W. Liang, M. Crump, F. Lin, Z. Zhu and A. K.-Y. Jen, Nano Energy, 2016, 22, 328-337.

29 C. Wang, C. Xiao, Y. Yu, D. Zhao, R. A. Awni, C. R. Grice, K. Ghimire, D. Constantinou, W. Liao, A. J. Cimaroli, P. Liu, J. Chen, N. J. Podraza, C.-S. Jiang, M. M. Al-Jassim, X. Zhao and Y. Yan, Adv. Energy Mater., 2017, 1700414.

30 A. Pisanu, C. Ferrara, P. Quadrelli, G. Guizzetti, M. Patrini, C. Milanese, C. Tealdi and L. Malavasi, J. Phys. Chem. C, 2017, 121, 8746-8751.

31 A. M. A. Leguy, Y. Hu, M. Campoy-Quiles, M. I. Alonso, O. J. Weber, P. Azarhoosh, M. van Schilfgaarde, M. T. Weller, T. Bein, J. Nelson, P. Docampo and P. R. F. F. Barnes, Chem. Mater., 2015, 27, 3397-3407.

32 V. L. Pool, B. Dou, D. G. Van Campen, T. R. Klein-Stockert, F. S. Barnes, S. E Shaheen, M. I. Ahmad, M. F. A. M. van Hest and M. F. Toney, Nat. Commun., 2017, 8, 14075.

33 M. Fanfoni and M. Tomellini, Il Nuovo Cimento D, 1998, 20, 1171-1182.

34 C. Eames, J. M. Frost, P. R. F Barnes, B. C. O'Regan, A. Walsh and M. S. Islam, Nat. Commun., 2015, 6, 7497.

35 N. Aristidou, C. Eames, I. Sanchez-Molina, X. Bu, J. Kosco, M. S. Islam and S. A. Haque, Nat. Commun., 2017, 8, 15218.

36 J. A. Dawson, A. J. Naylor, C. Eames, M. Roberts, W. Zhang, H. J. Snaith, P. G. Bruce and M. S. Islam, ACS Energy Lett., 2017, 2, 1818.

37 G. P. Nagabhushana, R. Shivaramaiah and A. Navrotsky, Proc. Natl. Acad. Sci. U. S. A., 2016, 113, 7717-7721.

38 F. Brivio, C. Caetano and A. Walsh, J. Phys. Chem. Lett., 2016, 7, 1083.

39 G. Kieslich, S. Sun and A. K. Cheetham, Chem. Sci., 2015, 6, 3430-3433.

40 W. Travis, E. N. K. Glover, H. Bronstein, D. O. Scanlon and R. G. Palgrave, Chem. Sci., 2016, 7, 4548-4556. 